\title{
Human immunodeficiency virus coinfection with drug-resistant Mycobacterium tuberculosis
}

\author{
Iringó Erzsébet Zaharia-Kézdi ${ }^{1 *}$, Carmen Chiriac ${ }^{1}$, Andrea Incze ${ }^{1}$, Nina Șincu ${ }^{1}$, Lilla Lorinczi ${ }^{2}$, Mihaela Patraulea ${ }^{3}$ \\ From The 9th Edition of the Scientific Days of the National Institute for Infectious Diseases Prof Dr Matei Bals \\ Bucharest, Romania. 23-25 October 2013
}

\section{Background}

Tuberculosis (TB), one of the oldest infectious diseases known to mankind, is a major cause of death for human immunodeficiency virus (HIV) infected people. Since 2008, the HIV/AIDS Mureş Regional Centre has been monitoring the increase in cases of HIV-TB with drugresistant mycobacteria (MTB). We have decided to analyze particular aspects of this phenomenon.

\section{Methods}

Retrospective cross-sectional study performed in the Infectious Diseases Clinic I Tîrgu Mureş during the period of 2008-2012. The study included HIV positive patients diagnosed with drug-resistant tuberculosis (TB-R). We monitored epidemiological and clinical data as well as the evolution of the disease. We compared the results with those of patients diagnosed during the period of 20032012 with HIV-drug-sensitive TB (TB-nR). For statistical analysis we used the Fisher and $\mathrm{T}$ tests.

\section{Results}

83 patients were diagnosed with HIV-TB-nR with an average age of 23 years. 36 were without antiretroviral therapy (ART), with a CD4 count of 198 cells $/ \mu \mathrm{L} ; 73 \%$ were adherent to ART, 32\% have deceased. 25 patients were diagnosed with HIV-TB-R (with an average age 25.6 years, over $50 \%$ were male) 7 of which were MDR, 11 pre-XDR, 7 XDR. Most cases of HIV TB-R were recorded in 2011, (8 cases of which 2 MDR, 6 pre-XDR). The patients suffered predominantly from secondary pulmonary $\mathrm{TB}$ (22) but also from extra pulmonary TB like meningoencephalitis (8). Resistance to isoniazid and rifampin was $100 \%$ (MDR), resistance to ethambutol $66.97 \%, 62.96 \%$ to streptomycin, $33.33 \%$ to kanamycin, $55.56 \%$ to quinolones. All strains maintained their sensitivity towards ethionamide, cycloserine, para-aminosalicylic acid. Hospitalization periods for patients with MDR were significantly longer (68 days) than those of patients without MDR (44 days): $\mathrm{T}$ test $\mathrm{p}=0.0045$. The adherence to ART of TB-R patients was much lower than that of TB-nR patients (odds ratio 2.12, Fisher test $\mathrm{p}=0.04$ ). We have not found significant statistical difference between $\mathrm{CD} 4$ lymphocyte numbers in MDR (140 cells/ $\mu \mathrm{L}$ ) and non-MDR patients. Drugresistance has had significant statistical influence over the mortality rate of patients (odds ratio, 3.11, Fisher test $\mathrm{p}=0.019$ ).

\section{Conclusion}

The incidence of HIV-TB-R is increasing with long hospitalization periods, difficult therapy, low adherence and high mortality rate.

\section{Authors' details}

${ }^{1}$ Infectious Diseases Clinic I, University of Medicine and Pharmacy Tîrgu Mureş, Romania. ${ }^{2}$ Department of Pneumophthisiology, University of Medicine and Pharmacy Tîrgu Mureş, Romania. ${ }^{3}$ Clinic of Pneumophtisiology, County Hospital Mureş, Romania.

Published: 16 December 2013

doi:10.1186/1471-2334-13-S1-08

Cite this article as: Zaharia-Kézdi et al:: Human immunodeficiency virus coinfection with drug-resistant Mycobacterium tuberculosis. BMC Infectious Diseases 2013 13(Suppl 1):O8.

\footnotetext{
* Correspondence: ezahariakezdi@yahoo.com

${ }^{1}$ Infectious Diseases Clinic I, University of Medicine and Pharmacy Tîrgu

Mureş, Romania

Full list of author information is available at the end of the article
} 